\title{
Effects of combining low-dose aspirin with a Chinese patent medicine on follicular blood flow and pregnancy outcome
}

\author{
YUE ZHAO $^{1}$, BOTAO DU ${ }^{1}$, XIAOYING JIANG ${ }^{2}$, MINGXING MA $^{1}$, LIQING SHI $^{1}$, \\ QINXI ZHANG ${ }^{1}$ and LILI ZHOU ${ }^{1}$ \\ ${ }^{1}$ Department of Reproductive Medicine, The Second Affiliated Hospital, Harbin Medical University, \\ Harbin, Heilongjiang 150086; ${ }^{2}$ The Fourth Ward of the Obstetrical Department, \\ Harbin Red Cross Central Hospital, Harbin, Heilongjiang 150000, P.R. China
}

Received August 18, 2013; Accepted February 28, 2014

DOI: $10.3892 / \mathrm{mmr} .2014 .2570$

\begin{abstract}
The aim of the present study was to investigate the clinical value of low-dose aspirin in combination with Tiao Jing $\mathrm{Cu}$ Yun pills in patients with polycystic ovary syndrome (PCOS) by measuring follicular peripheral blood flow parameters and the clinical efficacy. The study involved 78 infertile females with PCOS who were randomly divided into experimental $(n=38)$ and control $(n=40)$ groups. The subjects in the experimental group were treated with letrozole in combination with aspirin and Tiao Jing $\mathrm{Cu}$ Yun pills, and the control group was treated with letrozole alone. Transvaginal color Doppler ultrasonography was used to measure the endometrial thickness, pulsatility index (PI), resistance index (RI), peak systolic velocity and end diastolic velocity (EDV) of the follicular peripheral artery on the day of human chorionic gonadotropin administration. The patients who failed to become pregnant in the control group were reintegrated into the experimental group in the subsequent cycle and the clinical effect was observed. In the experimental group, subject perifollicular blood flow was more plentiful, and the PI and RI of the perifollicular blood flow were significantly reduced, while the EDV of the perifollicular blood flow and the rate of clinical pregnancy were markedly elevated compared with the subjects in the control group. Low-dose aspirin combined with Tiao Jing $\mathrm{Cu}$ Yun pills effectively improved perifollicular artery blood flow, and enhanced the oocyte quality and rate of clinical pregnancy.
\end{abstract}

\section{Introduction}

The incidence of polycystic ovary syndrome (PCOS) in females of childbearing age was 5-10\% in 2004 (1). With improvements

Correspondence to: Dr Botao Du, Department of Reproductive Medicine, The Second Affiliated Hospital, Harbin Medical University, 246 Xuefu Road, Nangang, Harbin, Heilongjiang 150086, P.R. China

E-mail: botaodu@163.com

Key words: polycystic ovary syndrome, aspirin, Tiao Jing $\mathrm{Cu}$ Yun pills, resistance index, pulsatility index in the research of PCOS, it has been identified that PCOS is one of the most common types of endocrine disorder in females (2). The incidence of infertility induced by ovulation disorders was $50-70 \%$ in 2010 (3). Therefore, drugs that promote ovulation have become a major treatment for patients with PCOS (4). A previous study reported that a favorable effect has been achieved following treatment with medicine, for example, letrozole that is used in clinics to promote ovulation, however, the ovulation rates were high and the pregnancy rates were low (5).

In order to investigate a method to improve the reactivity of the ovaries to ovulation medicines, as well as the egg quality and pregnancy outcomes, the present study was designed to observe the blood flow around the follicles of patients with PCOS following treatment with a low dose of aspirin in combination with Tiao Jing $\mathrm{Cu}$ Yun pills, and thus investigate the clinical value of this treatment.

\section{Patients and methods}

\section{Patients}

General information. A total of 78 patients with PCOS from the Department of Reproductive Medicine at The Second Affiliated Hospital of Harbin Medical University (Harbin, China) between March and December 2012 were selected for inclusion in the present study. The patients were of Chinese Han ethnicity from the Heilongjiang Province. The patients were randomly divided into two groups; the experimental group consisted of 38 patients with a mean age of $26.21 \pm 3.37$ years, body mass index of $24.60 \pm 4.24 \mathrm{~kg} / \mathrm{m} 2$ and duration of infertility of $2.09 \pm 1.14$ years. The control group consisted of 40 patients with a mean age of $26.30 \pm 3.38$ years, body mass index of $24.52 \pm 3.86 \mathrm{~kg} / \mathrm{m} 2$ and duration of infertility of $2.44 \pm 1.38$ years. No statistically significant differences between the two groups in age, body mass index, or duration of infertility were identified. The present study was approved by the ethics committee of The Second Affiliated Hospital, Harbin Medical University (Harbin, Heilongjiang, China). Written informed consent was obtained from the patients. The trial was registered in Heilongjiang Province Science and Technology Agency (No. LC08C19).

Diagnostic criteria. Due to the complicated heterogeneity and ethnic differences in the clinical manifestations of PCOS, the 
diagnostic criteria of PCOS has been continually disputed in individual countries and internationally, and three sets of criteria have been successively published (6-8). In July 2011, the Professional Committee of the Ministry of Health Medical Service Standard set the diagnostic criteria for PCOS in China, which is more suitable for the diagnosis and treatment of patients of a Chinese ethnicity. Suspected PCOS oligomenorrhea or irregular menstrual uterine bleeding are the necessary conditions for the diagnosis of PCOS. In addition, a patient may be diagnosed with suspected PCOS if they exhibit one of the following two symptoms: i) High androgen clinical manifestations or hyperandrogenism associated with high androgen levels; or ii) an ultrasound scan revealing a polycystic ovary, $\geq 12$ ovarian follicles with a diameter of 0.2-0.9 cm uni- or bilaterally, or an ovarian volume $\geq 10 \mathrm{~cm}^{3}$ [ovarian volume calculated according to the formula: $0.5 \mathrm{x}$ length $(\mathrm{cm}) \mathrm{x}$ width (cm) $x$ thickness $(\mathrm{cm})]$. To obtain a definitive diagnosis of PCOS, the symptoms must meet the suspected diagnosed conditions as well as the other potential causes of diseases associated with high androgen levels. Furthermore, ovulation disorders should be ruled out, including abnormal thyroid function, hyperprolactinemia, late onset adrenal cortical hyperplasia, 21-hydroxylase deficiency, adrenogenital syndrome, low ovarian reserve or premature aging of primary ovarian function, androgen-secreting ovarian or adrenal tumor and functional hypothalamic amenorrhea (8). All patients were aged between 22 and 30 years, sexually active and had experienced infertility for between one and five years.

Exclusion criteria. The exclusion criteria were as follows: Patients with a history of peptic ulcers or high blood pressure; patients without at least one oviduct unobstructed, as confirmed by iodolography; patients with acute genitourinary infections or sexually transmitted diseases; patients with serious genetic, physical or mental disorders; patients who used short-acting oral birth control pills for $\geq 3$ months to correct hormone levels; patients who used metformin for $\geq 3$ months due to insulin resistance or abnormal glucose tolerance test results; routine inspection of male semen of the partners of the patients during period of ovulation promotion identified abnormalities.

\section{Methods}

Grouping. The treatment for the experimental groups was as follows: Letrozole tablets (trade name, Winfrey; national medicine number, H19991001; Jiangsu Hengrui Medicine Co., Ltd., Lianyungang, China) were taken orally following five days of menstrual cramps or withdrawal bleeding at a dose of $2.5 \mathrm{mg}$ /day for five days. Also, 5-g Tiao Jing $\mathrm{Cu}$ Yun pills (national medicine number, Z20063997; Shaanxi Jun Bisha Pharmaceutical Co., Ltd., Xianyang, China), which regulate menstruation and promote pregnancy, were taken twice per day, every day until ovulation, along with enteric-coated aspirin tablets (national medicine number, J20080078; registered, H20090978; Bayer S.p.A., Milan, Italy) at a dose of $100 \mathrm{mg} /$ day until the day of ovulation.

The control group were treated as follows: Winfrey tablets were taken orally following five days of menstrual cramps or withdrawal bleeding at a dose of $2.5 \mathrm{mg} /$ day for five days. Patients who did not become pregnant were included in the experimental group in a subsequent cycle for ovulation treatment.

Monitoring. Transvaginal color Doppler ultrasonography (ALOKA ProSound Alpha 7; Hitachi Aloka Medical, Ltd. Tokyo, Japan) using a vaginal probe (frequency, $5 \mathrm{mHz}$ ) and 1-ml sample volume, was operated by the same person for each group and performed daily or every other day to monitor follicular growth, beginning at 10-12 days of menstruation. When the diameter of a follicle was $>14 \mathrm{~mm}$, the urine luteinizing hormone ( $\mathrm{LH})$ levels were checked every morning. When the diameter of at least one follicle was $\geq 18 \mathrm{~mm}$ and the level of urinary LH was positive $(+)$ or weakly positive $( \pm)$, 10,000 IU human chorionic gonadotropin (hCG) hormone was immediately injected intramuscularly to induce ovulation, with guided copulation for 24-36 h. On the day of the hCG injection, the number of follicles, vertical and horizontal diameters, endometrial thickness and the hemodynamics surrounding the dominant follicle were recorded. The sampling frame was placed around the follicular artery with the most evident color flow, and the perifollicular blood flow was measured following the appearance of at least three stable waveforms. Ovulation was monitored daily by ultrasound. Indications of mature follicle ovulation were as follows: The dominant follicles grew from $1.0 \mathrm{~cm}$ to $\geq 2.2 \mathrm{~cm}$ in diameter, followed by sudden disappearance, with surrounding echo enhancement. There was effusion in the posterior fornix. Then the echo-free zone in ovarian became fuzzy, with sparse dot- or cluster-like echoes, indicating formation of corpus luteum (9). The patients were administered with luteal support following ovulation, and the blood $\beta$-hCG was measured 14 days subsequently to determine whether the patient was pregnant, this is termed a biochemical pregnancy. After 28 days of ovulation, a vaginal ultrasound was performed as described above, to observe the gestational sac and fetal heart beat, which determined a clinical pregnancy.

Statistical analysis. SPSS statistical software, version 16.0 (SPSS, Inc., Chicago, IL, USA) was used for the statistical analysis. All data are presented as the mean \pm standard deviation and comparisons between groups were conducted using the independent samples t-test. The paired t-test was used to compare data prior to and following treatment; $\mathrm{P}<0.05$ was considered to indicate a statistically significant difference.

\section{Results}

Blood flow performance surrounding the follicles of the two different groups using Doppler ultrasonography. On the day of hCG injection, in the experimental group, the dominant follicles had strong echo signal of peripheral blood flow, with engorged perfusion and vigorous beating (Fig. 1). The patients in the control group exhibited a sparsely punctuated multi-display color flow signal or no color flow signals around the follicles (Fig. 2).

Endometrial thickness and clinical pregnancy rate in the two different groups. On the day of the hCG injection, the endometrial thickness was not identified to be significantly different between the two groups $(\mathrm{P}>0.05)$. The clinical pregnancy rate of the experimental group was significantly elevated compared with that of the control group. The significant difference is shown in Table I.

Perifollicular arterial blood flow correlation coefficient test results. Of the surrounding follicular flow parameters, the pulsatility index (PI), resistance index (RI) and end diastolic velocity (EDV) values in the experimental group were significantly 


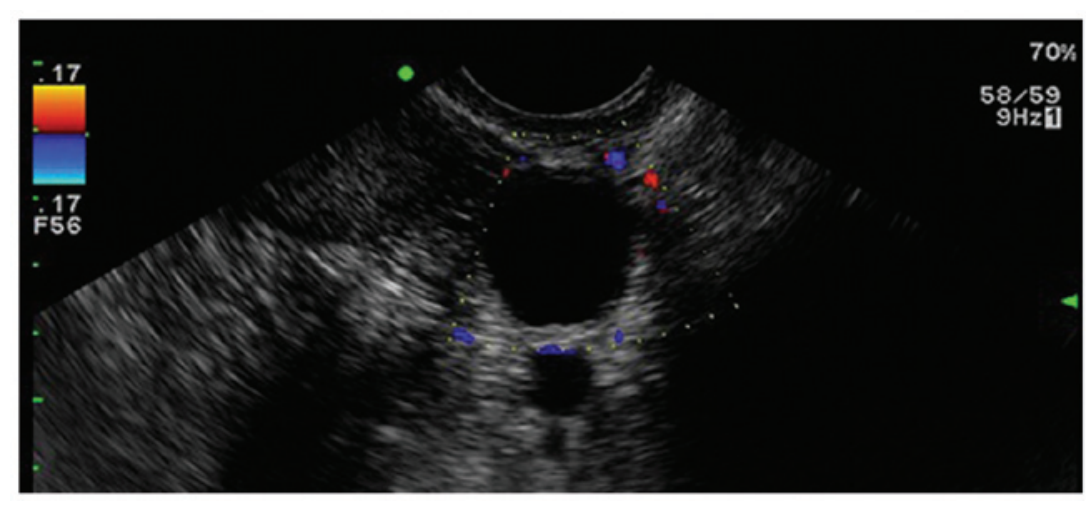

Figure 1. Representative image of the distribution of perifollicular blood flow in the experimental group.

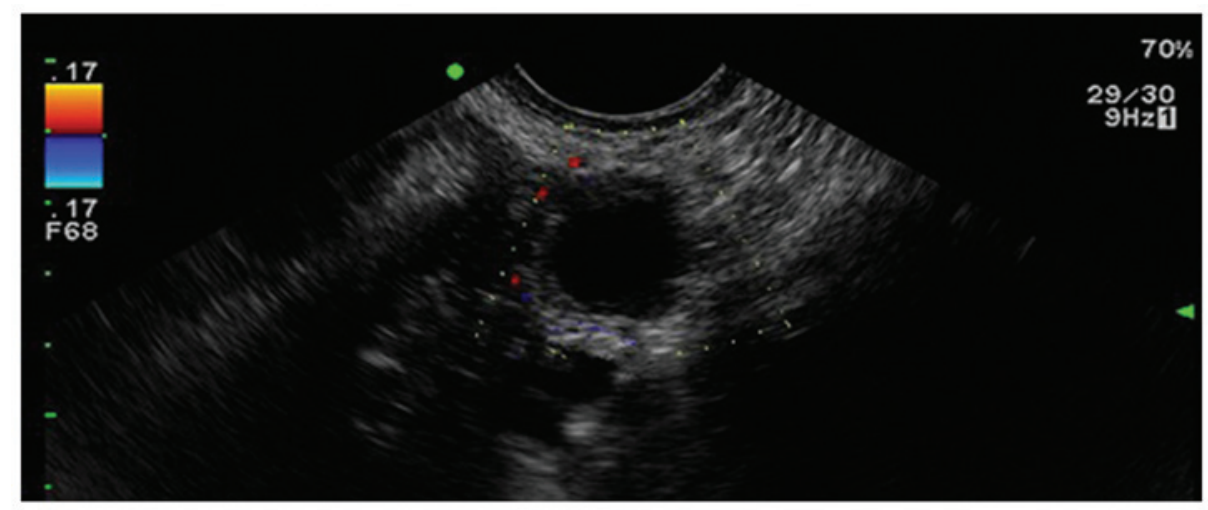

Figure 2. Representative image of the distribution of perifollicular blood flow in the control group.

different compared with those in the control group $(\mathrm{P}<0.01)$. No significant difference in the peak systolic velocity (PSV) values between the two groups was identified ( $P>0.05$; Table II).

The patients in the control group who did not become pregnant were reintegrated into the experimental group $(n=29)$, receiving ovulation treatment. The follicles were monitored on the day of HCG injection. Significant differences were identified in the perifollicular blood flow parameters PI and RI of the dominant follicles between prior to and following treatment with aspirin and Tiao Jing $\mathrm{Cu}$ Yun pills $(\mathrm{P}<0.01)$, with a significant difference in EDV values $(\mathrm{P}<0.05)$. However, no significant difference in the PSV values between prior to and following the treatment was observed ( $\mathrm{P}>0.05$; Table III).

Clinical outcome between the patients in the control group and those reintegrated into the experimental group. The control group pregnancy rate was only $27.50 \%$, while that of the experimental group of patients who were reintegrated in the second cycle was $37.93 \%$, which was significantly higher $(\mathrm{P}<0.05$; Table IV).

\section{Discussion}

Infertility is closely associated with intense social competition and the increasing pressures of life (10). Numerous patients make the uninformed decision to seek assisted reproductive technologies, including IVF treatment. However, the majority of these patients only require an agent for endocrine regulation
Table I. Endometrial thickness and clinical effect in the experimental and control groups on the day of human chorionic gonadotropin administration.

\begin{tabular}{lccc}
\hline Group & Cases & $\begin{array}{c}\text { Endometrial } \\
\text { thickness }(\mathrm{mm})\end{array}$ & $\begin{array}{c}\text { Clinical } \\
\text { pregnancy } \\
\text { rate }(\%)\end{array}$ \\
\hline Experimental & 38 & $9.376 \pm 1.187^{\mathrm{a}}$ & $42.11^{\mathrm{b}}$ \\
Control & 40 & $8.995 \pm 1.371$ & 27.50 \\
\hline
\end{tabular}

${ }^{\mathrm{a}} \mathrm{P}>0.05$ and ${ }^{\mathrm{b}} \mathrm{P}<0.05$ compared with the control group.

or a treatment that stimulates ovulation; fertility is achievable in certain patients simply by monitoring ovulation. PCOS is currently the most common cause of menstrual disorders in young females and it is the primary cause of ovulatory infertility (11). A high-quality mature egg is the most important factor for a successful pregnancy. At present, the predominant method of judging whether a follicle is mature involves measuring the size and morphological changes of the follicle by ultrasound and testing the plasma estrogen levels; judgment of follicle maturation by the morphology alone is not reliable. Therefore, a reliable and effective method is required (12-14).

In 1991, Kurjak et al (15) proposed that blood flow parameters surrounding the follicle could be used as an index 
Table II. Perifollicular blood flow parameters of the experimental and control groups on the day of human chorionic gonadotropin administration.

Perifollicular blood flow parameter

\begin{tabular}{lccccc}
\cline { 3 - 5 } Case & Cases & PI & RI & PSV & EDV \\
\hline Experimental & 38 & $0.898 \pm 0.874^{\mathrm{a}}$ & $0.60 \pm 0.546^{\mathrm{a}}$ & $16.423 \pm 2.077$ & $6.463 \pm 1.075^{\mathrm{a}}$ \\
Control & 40 & $1.027 \pm 0.944$ & $0.659 \pm 0.560$ & $16.825 \pm 2.858$ & $5.628 \pm 0.982$ \\
\hline
\end{tabular}

${ }^{a} \mathrm{P}<0.01$ compared with the control group. PI, pulsatility index; RI, resistance index; PSV, peak systolic velocity; EDV, end diastolic velocity. Data are expressed as the mean \pm standard deviation.

Table III. Comparison of perifollicular blood flow parameters prior to and following treatment.

\begin{tabular}{lcccc}
\hline & \multicolumn{4}{c}{ Perifollicular blood flow parameter } \\
\cline { 2 - 4 } Case & PI & RI & PSV & EDV \\
\hline Prior to & $0.970 \pm 0.085$ & $0.651 \pm 0.039$ & $16.727 \pm 1.978$ & $5.845 \pm 0.797$ \\
Following & $0.907 \pm 0.081^{\mathrm{a}}$ & $0.605 \pm 0.043^{\mathrm{a}}$ & $15.851 \pm 2.437$ & $6.228 \pm 0.813^{\mathrm{b}}$ \\
\hline
\end{tabular}

${ }^{\mathrm{a}} \mathrm{P}<0.01$ and ${ }^{\mathrm{b}} \mathrm{P}<0.05$ compared with prior to treatment (the values of the same subjects reintegrated from the control group to the experimental group). PI, pulsatility index; RI, resistance index; PSV, peak systolic velocity; EDV, end diastolic velocity.

Table IV. Clinical outcome in the new experimental and control groups.

\begin{tabular}{lcc}
\hline Group & Cases & Clinical pregnancy rate $(\%)$ \\
\hline New experimental & 29 & 37.93 \\
Control & 40 & 27.50 \\
\hline
\end{tabular}

${ }^{\mathrm{a}} \mathrm{P}<0.05$ compared with the control group.

to evaluate follicular growth, maturation and ovulation. The function status of a reproductive organ is closely associated with its angiogenesis and hemodynamic performance. The blood circulation status, amount of tissue blood perfusion and blood flow resistance are prerequisite conditions for the growth and development of ovarian follicles. In a previous study, it was demonstrated that following the injection of hCG, the values of the ovarian artery blood flow indexes, PI and RI, predict the development of follicles, and are reliable indicators for evaluating the degree of maturity of follicles and the quality of the ova (16). The present study indicated that the color signal surrounding the follicles was stronger in the group of patients taking letrozole alone compared with that of the group, which were treated with a combination of aspirin and Tiao Jing $\mathrm{Cu}$ Yun pills. This result indicated that the peripheral vascular formation of the follicles was increased, the liquid oxygen content of the follicles was rich and the conception rate was also high. Additionally, the present study demonstrated that the arterial blood flow index surrounding the follicle, presented as the values of PI and RI, was significantly higher compared with that of the experimental group. This result may be due to the fact that letrozole leads to increased synthesis and activity of follicle-stimulating hormone (FSH) through reducing estrogen synthesis. Due to the secretion of FSH, the concentrations of estradiol (E2) and progesterone in the serum rise, and the increasing concentration of progesterone antagonizes the vasodilation function of E2. The values of RI and PI of the follicular peripheral artery increase, which reduces the follicular blood perfusion rate and affects follicle maturation.

Aspirin has been used in the clinical setting for hundreds of years (17). The efficacy and safety of the application dose of aspirin used in infertility treatment remains controversial. Aspirin inhibits the activity of platelets, prevents the formation of microthrombi, reduces intravascular resistance and improves the local blood circulation (18). Low-dose aspirin (50-150 mg/day) reduces the tension of blood vessels, and improves tissue perfusion $(19,20)$, which increases the endometrial thickness, gland area, and activation of various female and progestational hormone receptors. Furthermore, aspirin improves the response to ovarian hormones, which improves the clinical pregnancy rate. The present study identified that, on the day hCG was injected, the perfusion of the peripheral artery of the dominant follicle increased, and the blood resistance of the PI, RI and PSV markedly decreased following treatment of PCOS with letrozole combined with aspirin, when compared with those of the control group. These changes are beneficial to the development and maturation of dominant follicles. In traditional Chinese medicine the kidneys are considered to be the key organs for the control of reproduction, the egg is the core of reproduction and fertility and is hidden in the kidney. Numerous Chinese traditional medicines are used to activate the blood circulation, nourish nerves, increase the blood flow around the ovaries and other 
endocrine glands, and induce the mature follicle to break away from the ovary. At the same time, the medicine is conducive to the implantation of a fertilized egg by improving the uterine blood supply and promoting the growth of the decidua.

The Tiao Jing $\mathrm{Cu}$ Yun pills contain numerous ingredients, including Astragalus, dodder, pilose antler, medlar, broiled Epimedium, Himalayan teasel root, parasitic Loranthus and Chinese yam. The pills have the function of tonifying the lddney and Yang, promoting blood circulation to restore menstrual flow, tuning up Chong and Ren, and aid with conception. Furthermore, the pills stimulate the ovaries and are involved in endocrine regulation, and promoting and inducing ovulation. Combined with suberect Spatholobus stem, Salvia miltiorrhiza, raspberry, poria cocos, Radix Paeoniae Rubra and Rhizoma Curculiginis, the pills are good for the kidney and spleen, and also promote blood circulation to restore menstrual flow and regulate menstrual function. The pill improves the function of the hypothalamic-pituitary-ovarian axis and stimulates the vitality of the ovaries (21). Tiao Jing $\mathrm{Cu}$ Yun pills have synergy with aspirin in promoting ovulation. It increases the ovulation rate and the probability of pregnancy by promoting ovulation and increasing the thickness of the endometrium, thus creating a favorable internal environment for implantation (22). The present study demonstrated that, on the day of the hCG injection, although the endometrial thickness was not significantly different in the group treated with lentrozole combined with aspirin and Tiao Jing $\mathrm{Cu}$ Yun pills compared with that of the control group, it was a suitable endometrial thickness (23). The results of the present study showed that the clinical pregnancy rate was $42.11 \%$ in the experimental group; however, in the control group it was only $27.50 \%$. The patients in the control group that had not become pregnant were integrated into the experimental group for the subsequent cycle and the clinical pregnancy rate was $37.93 \%$. This result indicated that letrozole combined with a small dose of aspirin and Tiao Jing $\mathrm{Cu}$ Yun pills results in an increased pregnancy rate in females with PCOS.

In conclusion, the promotion of menstruation via the combined use of low-dose aspirin, Tiao Jing $\mathrm{Cu}$ Yun pill and letrozole induces ovulation, follicular development and leads to pregnancy. A marked increase in clinical pregnancy rates was observed as a result of ovulation induction, which was significantly higher compared with using ovulation treatment. This method can replace conventional ovulation-induction treatments, which are associated with numerous side-effects, with the overall effect presenting as an improvement of the internal environment of the body. This treatment method is simple to use, affordable and the effect is significant; furthermore, it is a relatively safe and reliable infertility treatment option, worthy of promotion and application.

\section{References}

1. Archer JS and Chang RJ: Hirsutism and acne in polycystic ovary syndrome. Best Pract Res Clin Obstet Gynaecol 18: 737-754, 2004

2. Azziz R, Woods KS, Reyna R, Key TJ, Knochenhauer ES and Yildiz BO: The prevalence and features of the polycystic ovary syndrome in an unselected population. J Clin Endocrinol Metab 89: 2745-2749, 2004.
3. Hu WH, Qiao J, Wang LN and Tong J: Clinical features of the metabolic syndrome in patients with polycystic ovary syndrome. Beijing Da Xue Xue Bao 42: 159-163, 2010 (In Chinese).

4. Nahid L and Sirous K: Comparison of the effects of letrozole and clomiphene citrate for ovulation induction in infertile women with polycystic ovary syndrome. Minerva Ginecol 64: 253-258, 2012.

5. Nahid L and Sirous K: Comparison of the effects of letrozole and clomiphene citrate for ovulation induction in infertile women with polycystic ovary syndrome. Minerva Ginecol 64: 253-258, 2012.

6. Rotterdam ESHRE/ASRM-Sponsored PCOS consensus workshop group: Revised 2003 consensus on diagnostic criteria and long-term health risks related to polycystic ovary syndrome (PCOS). Human reprod 19: 41-47, 2004

7. Azziz R, Carmina E, Dewailly D, et al; Androgen Excess Society: Positions statement: criteria for defining polycystic ovary syndrome as a predominantly hyperandrogenic syndrome: an Androgen Excess Society guideline. J Clin Endocrinol Metab 91: 4237-4245, 2006.

8. Health industry standard of the People's Republic: Diagnosis of the polycystic ovary syndrome (WS 330-2011). The Ministry of Health Medical Service Standard Professional Committee of the People's Republic of China, 2011.

9. Zhang XN: Research on uterine and ovarian arterial blood flow for kidney-tonification and blood stasis-elimination treatment of polycystic ovarian syndrome. Chin J Pract Med 26: 864-867, 2010 (In Chinese).

10. Thessaloniki ESHRE/ASRM-Sponsored PCOS Consensus Workshop Group: Consensus on infertility treatment related to polycystic ovary syndrome. Hum Reprod 23: 462-477, 2008.

11. Goldenberg $\mathrm{N}$ and Glueck C: Medical therapy in women with polycystic ovarian syndrome before and during pregnancy and lactation. Minerva Ginecol 60: 63-75, 2008.

12. Borini A, Maccolini A, Tallarini A, Bonu MA, Sciajno R and Flamigni C: Perifollicular vascularity and its relationship with oocyte maturity and IVF outcome. Ann NY Acad Sci 943: 64-67, 2001.

13. Jokubkiene L, Sladkevicius P, Rovas L and Valentin L: Assessment of changes in volume and vascularity of the ovaries during the normal menstrual cycle using three-dimensional power Doppler ultrasound. Hum Reprod 21: 2661-2668, 2006.

14. Otsuki J, Okada A, Morimoto K, Nagai Y and Kubo H: The relationship between pregnancy outcome and smooth endoplasmic reticulum clusters in MII human oocytes. Hum Reprod 19: 1591-1597, 2004.

15. Kurjak A, Kupesic-Urek S, Schulman H and Zalud I: Transvaginal color flow Doppler in the assessment of ovarian and uterine blood flow in infertile women. Fertil Steril 56: 870-873, 1991.

16. Du B, Takahashi K, Ishida GM, Nakahara K, Saito H and Kurachi H: Usefulness of intraovarian artery pulsatility and resistance indices measurement on the day of follicle aspiration for the assessment of oocyte quality. Fertil Steril 85: 366-370, 2006.

17. Ozturk O, Greaves M and Templeton A: Aspirin dilemma. Remodelling the hypothesis from a fertility perspective. Hum Reprod 17: 1146-1148, 2002.

18. Saito H, Kaneko T, Kawachiya S, Du B, Ishida GM and Kurachi H: Effects of aspirin on the oocyte quality. Fertil Steril 76: S131, 2001.

19. Patrono C, García Rodríguez LA, Landolfi R and Baigent C: Low-dose aspirin for the prevention of atherosclerosis. N Engl J Med 353: 2373-2383, 2005.

20. Daya S: Is there a benefit of low-dose aspirin in assisted reproduction? Curr Opin Obstet Gynecol 18: 313-318, 2006.

21. Lai Q: 96 cases of clinical observation on Tiao Jing Cu Yun pills treatment for ovulation inhibition. Chin J Cosmetic Med 20: 353, 2011.

22. Xu ZM, Zhou YH and Peng JY: Curative effect observation on Tiao Jing $\mathrm{Cu}$ Yun pills for polycystic ovarian syndrome. Chin J Shanxi Trad Chi Med 27: 18-19, 2004 (In Chinese).

23. Isaacs JD Jr, Wells CS, Williams DB, Odem RR, Gast MJ and Strickler RC: Endometrial thickness is a valid monitoring parameter in cycles of ovulation induction with menotropins alone. Fertil Steril 65: 262-266, 1996. 